Planetary Systems in the Universe - Observation, Formation and Evolution

Proceedings IAU Symposium No. 202, (C)2004 IAU

Alan Penny, Pawel Artymowicz, Anne-Marie Lagrange, \& Sara Russell, eds.

\title{
Infrared spectroscopic search for short-period giant extrasolar planets
}

\author{
Günter Wiedemann
}

European Southern Observatory, 85748 Garching, FRG

L. Drake Deming, Gordon L. Bjoraker, Cedric Goukenleuque

NASA/GSFC, Greenbelt, Md 20771, USA

\begin{abstract}
IR spectroscopy with a resolution $\lambda / \Delta \lambda \gtrsim 10,000$ is a powerful technique for the investigation of short-periodic giant extra-solar planets. For an unambiguous direct detection attempt one exploits the large-amplitude Doppler modulation of the planet's IR spectrum. A successful measurement of the planet's radial velocity amplitude would yield directly the planet-star mass ratio. Spectral information can be extracted if high per-pixel $\mathrm{S} / \mathrm{N}$ levels are achieved.
\end{abstract}

\section{Introduction}

High-resolution spectrographs at large telescopes are employed to search for the intrinsic infrared spectrum emitted by a planetary atmosphere.

At a distance from Earth of several tens of parsec, a 'hot Jupiter' in a 4-day orbit, corresponding to an orbital distance of ca. $0.05 \mathrm{AU}$, and its parent star are spatially inseparable. The planet's spectrum is unavoidably superimposed on that of the star, resp. its continuum. The planet's relative IR brightness is of order $10^{-4}$ or less. A detection at this level is usually impossible from the ground. One can, however, exploit the natural signal modulation provided by the planetary system itself for a 'synchronous detection': during its orbit, a planet's spectrum is periodically Doppler-shifted with a velocity amplitude determined by the usually unknown orbital inclination and the orbital velocity. The latter is known for planets of stars with established reflex motions. The Doppler-shift of the planet's spectrum is greater than that of the star by the starto-planet mass ratio, i.e. typ. 1000 times. For all but the smallest inclination angles, the velocity amplitude is large compared to fluctuations in stellar and atmospheric absorption lines, and easily resolvable with a high-resolution IR spectrograph. Direct planet searches are carried out predominantly in the IR where the important molecules $\mathrm{CH}_{4}$ and $\mathrm{CO}$ have rich line spectra.

\section{Technique and Targets}

The search for a planet near a candidate star requires a time series of stellar spectra, spanning several orbital periods of the planet. The planetary contribution is distinguished by its unique temporal behavior, i.e. the periodic global 
Keplerian frequency shift. The phase and the period of this shift are known for targets with established radial velocity planets.

Potential targets for the direct velocity search technique are all giant gas planets in close orbits around dwarf or subgiant stars. The notion of gas atmospheres on giant extrasolar planets is now widely accepted. Observational evidence for at least one object is provided by the mean density of $0.4 \mathrm{~g} / \mathrm{ccm}$ of the HD209458 planet, derived from the size and mass determination during its transit (Charbonneau et al. 1999).

The presence of a rich stable line spectrum in the parent star is not required for the direct planet detection, although it helps to provide a velocity reference. For practical reasons such as the substantial amounts of observing time required at large telescopes, and the scarcity of suitable IR spectrographs worldwide, direct searches will likely be limited to stars with known 'RV planets'. This may change if in the future daytime observing is implemented at large telescopes, setting free telescope time for bright star IR observations.

The reasons for the limitation to short-period orbits are: the large planetary radial velocities, the IR brightness of planets close to their stars, and the possibility to observe a planet over several orbital periods in a reasonable time.

Of the nearly $50 \mathrm{RV}$ planets known at this time (end 2000), ca $25 \%$ have orbital periods of 2 weeks or less, and are potential candidates for a direct IR search.

\section{Observations and Analyses}

This observing program began in 1997, using the CSHELL spectrograph on Mauna Kea (Greene et al. 1993) and the PHOENIX instrument (Hinkle et al. 1998) on Kitt Peak. Exploratory observations were targeting 51 Peg and Ups And. The most intensely observed system has been $\tau$ Boo (F6V). A deep search for a methane signature in the IR spectrum of $\tau$ Boo was made at the IRTF on 29 March -3 April 1998. About 1200 spectral frames were acquired under good to excellent observing conditions. Two complete orbits of the planet were covered, with a total on-source intergration time of 31 hours.

The choice of the $3.3 \mu \mathrm{m} \nu 3$-band of methane for these observation reflects a necessary compromise between available instrument capabilities, stellar noise flux, terrestrial thermal background emission and expected planet line strengths and multiplicity. Methane is indeed a sensible diagnostic, with considerable line strengths even at the low relative abundance (Fegley \& Lodders 1996, Burrows $\&$ Sharp 1999) at the high temperature of $\tau$ Boo $b$ of $\approx 1600 K$. Increasing the $\mathrm{CH}_{4}$ abundance in model spectra can actually lead to overlapping of saturated lines and hence reduced detectable contrast.

The analysis mainly consists of the search for a singular Doppler-modulated spectrum with an a priori unknown velocity amplitude. For complex methane spectra in a small spectral interval, cross-correlation with a synthetic template spectrum is the preferred method. Planet model atmospheres for the computation of template spectra are already available ( e.g. Burrows \& Sharp 1999) and are being further developed. Details on the methane search on $\tau$ Boo b have been reported (Wiedemann, Deming \& Bjoraker 2001). While no convincing 
detection has been achieved, the occurrence of confusion-limited, but significant -i.e. above the photon noise- peaks in the correlation function near a velocity amplitude of $71 \mathrm{~km} / \mathrm{sec}$, clearly shows the path to future observations and improved analyses, including phase-dependent intensities.

\section{VLT observations of HD $\mathbf{7 5 2 8 9}$}

Substantially better observations are possible with large IR array spectrographs at 8-10 $\mathrm{m}$ telescopes. The program presented here has used the ISAAC spectrograph (Moorwood 1997) at the ESO VLT for observations of the first known short-period (3.5 d) planet star HD75289 (G0 V), with a probable sub-Jupiter mass of $\mathrm{m} \times \sin \mathrm{i}=0.42 \mathrm{~m}_{J}$ (Udry et al. 2000). Bright star observations repeated over several orbital periods of the planets are greatly helped by the service observing mode implemented at the VLT. At the expense of reduced control over the data recording, the 'observer' is not concerned with weather problems or month-long visits to the observatory. The quality of the data is virtually guaranteed within the requirements specified in the observing preparation phase. Experience has shown, however, that substantial efforts have to be spent on instrument calibration and data reduction, if the $\mathrm{S} / \mathrm{N}$ values permitted by the sheer photon noise are to be approached in practice. Improved observing and calibration procedures were developed after the first successful run. HD75289 observations were carried out in bright time over a 6-week period in April and May 2000. Over 700 individual spectra were recorded, distributed over the velocity phases, and with a total accumulated on-chip integration time of more than 21 hours. During the data reduction, previously unknown resp. neglected instrumental and atmospheric effects were discovered, for which correction procedures had to be developed. The analysis is expected to be complete in early 2001. The first HD75289 data were recorded in the $2.3 \mu \mathrm{m}$ range which includes the first overtone $\mathrm{CO}$ ro-vibration transitions. $\mathrm{CH}_{4}$ observations of HD75289 have been scheduled for early 2001 .

\section{References}

Burrows, A., \& Sharp, C.M. 1999, ApJ, 512, 843

Charbonneau, D., Brown, T.M., Latham, D.W., \& Mayor, M. 2000, ApJ, 529, L45

Fegley, B. Jr., \& Lodders, K. 1996, ApJ, 472, L37

Greene, T.P., Tokunaga, A.T, Toomey, D.W., \& Carr J.S. 1993, Proc. SPIE, 1946, 313

Hinkle, K.H., Cuberly, R.W., Gaughan, N.A., Heynssens, J.B., Joyce, R.R., Ridgway, S.T., Schmitt, P., \& Simmons, J.E. 1998, Proc SPIE. 3354, 810

Moorwood, A.F.M. 1997, Proc. SPIE, 2871, 1146

Udry, S., Mayor, M., Naef, D., Pepe, F., Queloz, D., Santos, N. C., Burnet, M., Confino, B., \& Melo, C. 2000, A\&A, 356, 590

Wiedemann, G., Deming, L.D., \& Bjoraker, G. 2001, ApJ, 546, in press 